\title{
openheart Risk classification in primary prevention of CVD according to QRISK2 and JBS3 'heart age', and prevalence of elevated high-sensitivity $C$ reactive protein in the UK cohort of the EURIKA study
}

\author{
leuan Johns, ${ }^{1}$ Konstantinos E Moschonas, ${ }^{2}$ Jesús Medina, ${ }^{3}$ \\ Nicholas Ossei-Gerning, ${ }^{4}$ George Kassianos, ${ }^{5}$ Julian P Halcox ${ }^{6}$
}

\begin{abstract}
- Additional material is published online only. To view please visit the journal online (http://dx.doi.org/10.1136/ openhrt-2018-000849).
\end{abstract}

To cite: Johns I, Moschonas KE, Medina J, et al. Risk classification in primary prevention of CVD according to QRISK2 and JBS3 'heart age', and prevalence of elevated high-sensitivity $C$ reactive protein in the UK cohort of the EURIKA study. Open Heart 2018;5:e00849. doi:10.1136/ openhrt-2018-000849

Received 20 May 2018 Revised 2 August 2018 Accepted 31 August 2018

Check for updates

(C) Author(s) (or their employer(s)) 2018. Re-use permitted under CC BY-NC. No commercial re-use. See rights and permissions. Published by BMJ.

For numbered affiliations see end of article.

Correspondence to

Professor Julian P Halcox; J.P.J. Halcox@swansea.ac.uk

\section{ABSTRACT}

Objectives This study assessed cardiovascular disease (CVD) risk classification according to QRISK2, JBS3 'heart age' and the prevalence of elevated high-sensitivity C reactive protein (hsCRP) in UK primary prevention patients. Method The European Study on Cardiovascular Prevention and Management in Usual Daily Practice (EURIKA) (NCT00882336) was a cross-sectional study conducted in 12 European countries. 673 UK outpatients aged $\geq 50$ years, without clinical CVD but with at least one conventional CVD risk factor, were recruited. 10-year CVD risk was calculated using QRISK2. JBS3 'heart age' and hsCRP level were assessed according to risk category. Results QRISK2 and JBS3 heart age was calculated for 285 of the 305 patients free from diabetes mellitus and not receiving a statin. QRISK2 classified $28 \%, 39 \%$ and $33 \%$ of patients as low $(<10 \%)$, intermediate $(10 \%$ to $<20 \%$ ) and high ( $\geq 20 \%$ ) risk, respectively. Two-thirds of low-risk patients and half of intermediate-risk patients had a heart age $\geq 5$ years and $\geq 10$ years higher than their chronological age, respectively. Half of low-risk patients had hsCRP levels $\geq 2 \mathrm{mg} / \mathrm{L}$ and approximately $40 \%$ had levels $\geq 3 \mathrm{mg} / \mathrm{L}$. Approximately $80 \%$ of low-risk patients had both elevated hsCRP and heart age relative to their chronological age.

Conclusions Almost 40\% more patients in this 'at risk' group would be eligible for statin therapy following the lowering of the National Institute for Health and Care Excellence treatment threshold to $\geq 10 \% 10$-year risk. Of patients falling below this treatment threshold, almost al were at increased lifetime risk as measured by JBS3, and of these, the majority had elevated hsCRP levels. These patients with high absolute risk may benefit from early primary CVD prevention.

\section{INTRODUCTION}

Cardiovascular disease (CVD) is a major health concern. It is the leading cause of mortality in Europe and accounts for a third of all deaths in the UK. ${ }^{12}$ Multiple interacting

\section{Key messages}

What is already known about this subject?

- Novel risk metrics such as JBS3 'heart age' may identify patients with modest conventional cardiovascular risk scores who are at elevated lifetime risk.

- Patients with elevated levels of high-sensitivity $\mathrm{C}$ reactive protein are recognised to be at increased cardiovascular risk, and results of the recent CANTOS trial suggest clinical benefit in treating those with low-grade inflammation.

What does this study add?

- An additional $~ 40 \%$ of patients in this 'at risk' study cohort would be eligible for lipid-lowering therapy following the reduction of the National Institute for Health and Care Excellence treatment threshold from $\geq 20 \%$ to $\geq 10 \% 10$-year cardiovascular disease (CVD) risk.

- A considerable proportion of those falling below this treatment threshold had an increased JBS3 'heart age' as well as elevated levels of high-sensitivity C reactive protein

How might this impact on clinical practice?

- Measurement of high-sensitivity C reactive protein in addition to the use of lifetime risk metrics may identify a substantial proportion of patients at greater absolute long-term cardiovascular risk than expected on the basis of their conventional 10-year risk scores. These individuals may benefit more from earlier intensive CVD prevention measures.

risk factors contribute to the development of CVD. ${ }^{3}$ Well-established risk factors include old age, male sex, smoking, elevated cholesterol levels, hypertension and diabetes mellitus. ${ }^{3}$

Cardiovascular risk assessment is routinely performed to assess an individual's risk of 
CVD events and to guide preventive treatment strategies. The most frequently used risk algorithm is the QRISK2 risk assessor, which is calibrated using UK patient data and assesses an extensive array of risk factors, incorporating body mass index (BMI), ethnicity, an area of residence-based deprivation index, type 2 diabetes mellitus, antihypertensive treatment status, family history of CVD, chronic kidney disease, atrial fibrillation and rheumatoid arthritis into the algorithm. ${ }^{4}$ This method provides an estimation of the 10-year risk of experiencing a CVD event and is widely used in the primary care setting. ${ }^{5}$ The JBS3 risk calculator released in the Joint British Societies' CVD prevention guidelines in $2014^{6}$ was developed from the QRISK2 lifetime cardiovascular risk algorithm. JBS3 provides novel lifetime risk metrics including 'heart age', which indicates the age of an individual of the same gender and ethnicity with an equivalent annual risk of a cardiovascular event but with an optimal risk factor profile. $^{6}$

Assessment of global cardiovascular risk is recommended for identification of patients likely to benefit most from medical intervention as part of CVD primary prevention. ${ }^{356}$ European guidelines for CVD prevention recommend concurrent assessment of both cholesterol levels and 10-year CVD risk when considering commencing antihypertensive or lipid-lowering therapy in apparently healthy, asymptomatic individuals. ${ }^{3}$ National Institute of Health and Care Excellence (NICE) guidance on CVD risk assessment and lipid modification published in 2014 lowered the threshold for initiation of lipid-lowering therapy from $\geq 20 \% \quad 10$-year CVD risk to include individuals at $\geq 10 \%$ risk, potentially greatly increasing the number eligible for statin treatment in primary CVD prevention. ${ }^{5}$

In addition to established CVD risk factors, patients with elevated plasma levels of inflammatory biomarkers, such as high-sensitivity $\mathrm{C}$ reactive protein (hsCRP), are recognised to be at increased cardiovascular risk. ${ }^{7}$ A continuous association exists between CRP level and risk of cardiovascular events, ${ }^{8}$ remaining significant after adjustment for conventional risk factors. ${ }^{7910}$ Furthermore, inclusion of hsCRP data into risk models enhances the predictive value of established risk factors. ${ }^{911} 12$ Measurement of hsCRP may therefore be useful in identifying individuals at higher risk of cardiovascular risk than predicted by traditional risk algorithms, although not used routinely in the UK and Europe. Improving the accuracy of CVD risk assessment remains a major priority in the primary prevention of CVD given that one-fifth or more of all cardiovascular events occur in those without major conventional CVD risk factors, ${ }^{13}$ and half of all myocardial infarctions and strokes occur in individuals with a lipid profile which might not warrant therapeutic intervention under established guidelines. ${ }^{14}$ The JUPITER study and more recent CANTOS study have shown that both rosuvastatin $20 \mathrm{mg}$ daily and treatment with a novel anti-interleukin 1 monoclonal antibody can improve clinical outcomes in those patients with evidence of low-grade inflammation. ${ }^{14} 15$
We conducted a post hoc analysis of the UK cohort of the European Study on Cardiovascular Risk Prevention and Management in Usual Daily Practice (EURIKA; ClinicalTrials.gov identifier: NCT00882336), a cross-sectional study conducted to assess the management of cardiovascular risk factors in primary care in Europe. ${ }^{16}$ Our study explored the potential clinical impact of using more contemporary CVD risk assessment strategies risk for primary prevention in a representative real-world patient population and how introduction of the recent NICE guidance criteria for lipid modification ${ }^{5}$ might further change clinical decision-making. We first assessed CVD risk assessment according to QRISK2 (2017) in those without diabetes and not already commenced on lipid-lowering therapy. Second, we considered the potential of 'heart age', an indicator of lifetime risk, in further guiding preventative strategies in these patients. We also assessed the prevalence of elevated hsCRP levels in relation to CVD risk classification.

\section{METHOD}

\section{Study design and participants}

The EURIKA study (ClinicalTrials.gov identifier: NCT00882336) was a cross-sectional study conducted in 12 European countries (Austria, Belgium, France, Germany, Greece, Norway, Russia, Spain, Sweden, Switzerland, Turkey and the UK). The study protocol has been described in detail elsewhere. ${ }^{16}$ In brief, approximately 60 physicians were randomly selected from each country using the OneKey Healthcare Professional Database and were stratified by age, sex and specialty. Physicians from specialties involved in cardiovascular disease risk control were considered for selection. This included primary care physicians, cardiologists, endocrinologists and internal medicine specialists. Patients attending outpatient appointments who met the selection criteria (online supplementary appendix 1) were invited consecutively to participate in the study. Patients recruited were at least 50 years of age and free from clinical CVD, but with at least one classical risk factor (dyslipidaemia, hypertension, smoking, diabetes mellitus or obesity). Hypertension was defined as blood pressure $\geq 140 / 90 \mathrm{~mm} \mathrm{Hg}$ and $\geq 130 / 80 \mathrm{~mm} \mathrm{Hg}$ for those with diabetes mellitus. Patients receiving antihypertensive medication following a previous diagnosis of hypertension were also considered hypertensive. Dyslipidaemia was defined as total cholesterol $\geq 5 \mathrm{mmol} / \mathrm{L}$ or low-density lipoprotein (LDL)-cholesterol $\geq 4.5 \mathrm{mmol} / \mathrm{L}$. Similarly, patients with a prior diagnosis of dyslipidaemia who were receiving lipid-lowering therapy were also classed as having dyslipidaemia. Diabetics were considered to be dyslipidaemic if total cholesterol $\geq 4.5 \mathrm{mmol} / \mathrm{L}$ or LDL-cholesterol $\geq 2.5$ $\mathrm{mmol} / \mathrm{L}^{17}$

Patient information was gathered via clinical records, physical examination and a 12-hour fasting blood sample, which was collected within 1 day of the outpatient consultation. Laboratory analyses were conducted in a centralised laboratory. ${ }^{16}$ Additionally, a patient-specific 
questionnaire was used to collect sociodemographic data and information relating to CVD risk factors and current medications.

A total of 7641 patients were enrolled in the study across Europe. Six hundred seventy-three UK patients were selected by 69 participating UK physicians. In the present analysis, only patients with hsCRP levels available were considered $(672 / 673)$. Subjects with diabetes mellitus (already considered at risk regardless of hsCRP level) and those already receiving a statin (for whom a treatment decision has already been made) were also excluded from analysis. Three hundred five patients were free from diabetes mellitus and not receiving a statin, of whom data were available for calculation of QRISK2 CVD risk score in 285.

\section{Conventional cardiovascular risk classification}

Participants' 10-year cardiovascular risk was initially assessed by the conventional CVD risk calculator QRISK2 (2017 version). ${ }^{4}$ QRISK2 risk categories were $<10 \%$ low risk, $10 \%$ to $<20 \%$ intermediate risk and $\geq 20 \%$ high risk.

\section{JBS3 heart age}

Heart age was calculated using the JBS3 online risk assessment tool ${ }^{18}$ between 26 April and 11 May 2016. The difference between heart age and chronological age was calculated. The JBS3 algorithm could model a maximum heart age of 95 years. A total 53 subjects had heart ages exceeding this upper limit when the algorithm was applied. For the purpose of estimation of heart age, averages, differences between heart age and chronological age, and classification, these subjects were considered as having a heart age of 95 years.

\section{Assessment of hsCRP levels}

CRP levels were measured in a central core laboratory by a high-sensitivity immunoturbidimetry method (Roche P-Modular). HsCRP levels were categorised according to cardiovascular risk category. The thresholds for classifying hsCRP levels were set according to both American Heart Association/Centers for Disease Control and Pguidelines (those with hsCRP $<1 \mathrm{mg} / \mathrm{L}$ should be considered low risk, hsCRP 1 to $<3 \mathrm{mg} / \mathrm{L}$ as intermediate and $\geq 3 \mathrm{mg} / \mathrm{L}$ as high risk), ${ }^{7}$ and also according to JUPITER study selection criteria (those with hsCRP $>2 \mathrm{mg} / \mathrm{L}$ would have been eligible for recruitment) ${ }^{14}$ HsCRP levels were available for all 285 for whom QRISK2 CVD risk could be calculated.

\section{Statistical analysis}

Statistical analysis was carried out using IBM SPSS Statistics V.20.

\section{RESULTS}

\section{Demographics and baseline characteristics}

Subject demographics and baseline characteristics for the entire UK cohort, those free from diabetes mellitus and not receiving a statin, are shown in table 1.

Table 1 Baseline characteristics for the entire UK cohort, for patients free from diabetes and not receiving astatin, and according to QRISK2 (2017) 10-year cardiovascular risk

\begin{tabular}{|c|c|c|c|c|c|}
\hline & Overall ( $\mathrm{N}=673$ ) & $\begin{array}{l}\text { Patients without } \\
\text { DM who were not } \\
\text { receiving statin } \\
\text { treatment }(\mathrm{N}=305)\end{array}$ & $\begin{array}{l}\text { Low risk }(<10 \%) \\
(\mathrm{N}=80)\end{array}$ & $\begin{array}{l}\text { Intermediate risk } \\
(10 \% \text { to }<20 \%) \\
(N=110)\end{array}$ & $\begin{array}{l}\text { High risk } \\
(\geq 20 \%) \\
(\mathrm{N}=95)\end{array}$ \\
\hline Age, years & $65.0(8.9)$ & $64.4(9.4)$ & $55.6(4.6)$ & $62.5(5.6)$ & $72.7(6.9)$ \\
\hline Men, n (\%) & $344(51.1)$ & $135(44.3)$ & $19(23.8)$ & $55(50)$ & $54(56.8)$ \\
\hline Dyslipidaemia, $n(\%)$ & $357(53.0)$ & 68 (22.3) & $21(26.3)$ & $23(20.9)$ & $19(20.0)$ \\
\hline Total cholesterol, mmol/L & $5.0(1.2)$ & $5.7(1.0)$ & $5.7(0.9)$ & $5.7(1.1)$ & $5.7(1.0)$ \\
\hline LDL-C, mmol/L & $2.9(1.0)$ & $3.5(0.8)$ & $3.4(0.7)$ & $3.5(0.9)$ & $3.5(0.9)$ \\
\hline $\mathrm{HDL}-\mathrm{C}, \mathrm{mmol} / \mathrm{L}$ & $1.4(0.4)$ & $1.5(0.4)$ & $1.6(0.5)$ & $1.5(0.4)$ & $1.5(0.4)$ \\
\hline Triglycerides, $\mathrm{mmol} / \mathrm{L}$ & $1.7(0.9)$ & $1.6(0.9)$ & $1.4(0.6)$ & $1.6(0.8)$ & $1.7(1.1)$ \\
\hline Hypertension, n (\%) & $481(71.5)$ & $223(73.1)$ & $44(55.0)$ & $84(76.4)$ & $84(88.4)$ \\
\hline SBP, $\mathrm{mm} \mathrm{Hg}$ & $136.3(15.9)$ & $137.2(15.6)$ & $128.7(13.5)$ & $137.8(14.7)$ & $143.9(14.6)$ \\
\hline $\mathrm{DBP}, \mathrm{mm} \mathrm{Hg}$ & $79.1(9.9)$ & $80.1(10.1)$ & $80.5(9.3)$ & $82.3(10.1)$ & $78.3(9.8)$ \\
\hline $\mathrm{DM}, \mathrm{n}(\%)$ & $152(22.6)$ & $0(0)$ & $0(0)$ & $0(0)$ & $0(0)$ \\
\hline $\mathrm{BMI}, \mathrm{kg} / \mathrm{m}^{2}$ & $29.3(6.4)$ & $28.6(6.2)$ & $28.9(6.2)$ & $29.1(5.1)$ & $28.5(4.9)$ \\
\hline Obese, n (\%) & $239(35.5)$ & 97 (31.8) & $31(38.8)$ & 35 (31.8) & $26(27.4)$ \\
\hline Current smoker, n (\%) & $110(16.3)$ & $56(18.4)$ & $40(50.0)$ & 49 (44.5) & $52(54.7)$ \\
\hline HsCRP, mg/L, median (IQR) & $2.1(3.4)$ & $2.4(3.6)$ & $2.1(4.7)$ & $2.8(4.1)$ & $2.3(2.7)$ \\
\hline
\end{tabular}

Data are mean (SD) unless otherwise indicated.

$\mathrm{BMI}$, body mass index; DBP, diastolic blood pressure; DM, diabetes mellitus; HDL-C, high-density lipoprotein cholesterol; hsCRP, highsensitivity C-reactive protein; LDL-C, low-density lipoprotein cholesterol; SBP, systolic blood pressure. 


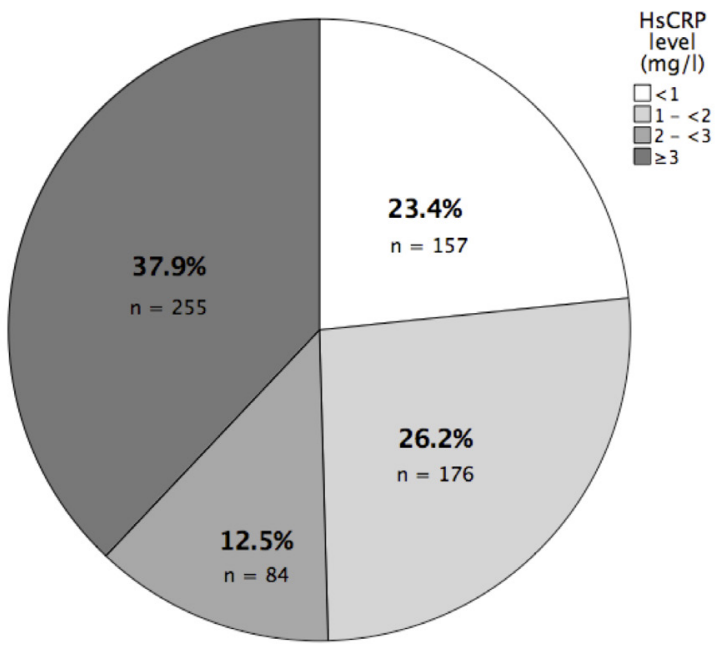

Figure 1 High-sensitivity $\mathrm{C}$ reactive protein (hsCRP) levels in the total UK cohort.

The mean age of the entire cohort was 65 years and $51.1 \%$ were men. Over $22.6 \%$ (152) had diabetes mellitus, approximately $70 \%$ were hypertensive, over half had dyslipidaemia and approximately $35 \%$ were obese (mean BMI $\sim 30 \mathrm{~kg} / \mathrm{m}^{2}$ ). The baseline characteristics of subjects without DM and not receiving a statin were otherwise very similar to the overall cohort, except a lower proportion had a prior diagnosis of dyslipidaemia (22.3 vs $53.0 \%$; $\mathrm{p} \leq 0.0001)$.

\section{HsCRP levels}

HsCRP levels were available for 672 out of 673 of the overall UK study group and 304 out of the 305 patients without diabetes who were not receiving statin treatment. The median hsCRP level for the overall UK population was $2.1 \mathrm{mg} / \mathrm{L}$. The 305 patients without diabetes who were not receiving statins had a median hsCRP of 2.4 $\mathrm{mg} / \mathrm{L}$. Approximately half of all the UK patients had hsCRP levels $\geq 2 \mathrm{mg} / \mathrm{L}$ and over one-third of all patients had hsCRP $\geq 3 \mathrm{mg} / \mathrm{L}$ (figure 1).

\section{QRISK2 10-year CVD risk}

The median 10-year QRISK2 score was 15\% (IQR 13.85). QRISK2 classified $28 \%(\mathrm{n}=80)$ of patients as low $(<10 \%)$, $39 \%(\mathrm{n}=110)$ as intermediate $(10 \%$ to $<20 \%)$ and $33 \%$ $(\mathrm{n}=95)$ as high $(>20 \%)$ 10-year CVD risk.

\section{HsCRP levels according to QRISK2 category}

HsCRP levels in relation to QRISK2 category are shown in figure 2. In the subgroup of patients without diabetes and not receiving statin treatment, $41.4 \%$ had hsCRP levels $\geq 3 \mathrm{mg} / \mathrm{L}$. Moreover, $\geq 50 \%$ of patients in each risk category had hsCRP levels $\geq 2 \mathrm{mg} / \mathrm{L} ; 41.3 \%$ of low-risk patients had a hsCRP $\geq 3 \mathrm{mg} / \mathrm{L}$ and $50.0 \%$ had a hsCRP $\geq 2 \mathrm{mg} / \mathrm{L}$.

\section{Heart age}

Heart ages expressed in relation to chronological age according to QRISK2 are displayed in figure 3 (online

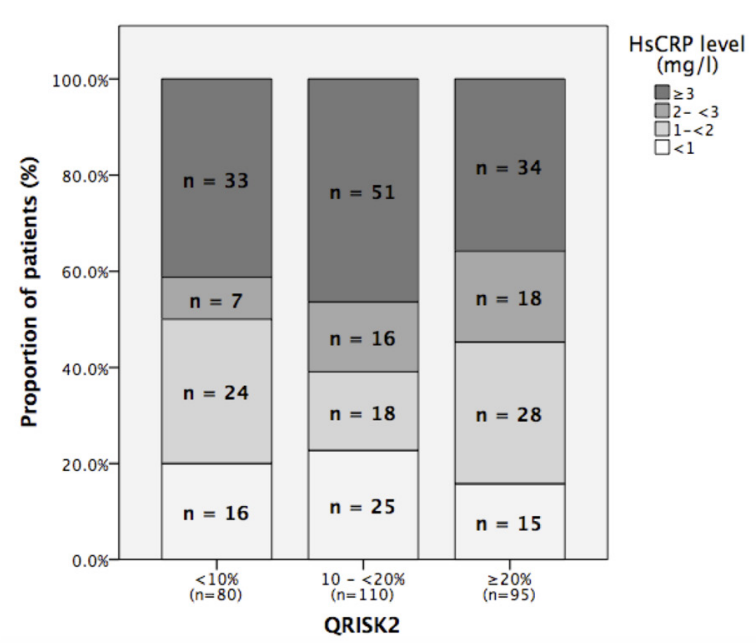

Figure 2 High-sensitivity $\mathrm{C}$ reactive protein (hsCRP) levels according to cardiovascular disease risk as calculated by QRISK2 in patients free from diabetes and not receiving a statin.

supplementary table 1) for the 285 patients without diabetes or taking a statin. Their median heart age was 73.0 years (IQR 18.5 years), and they had a median heart age 10.0 years greater than their chronological age. The heart age of all but two of these patients $(>99 \%)$ exceeded their chronological age, of whom $16.3 \%(n=46)$ had a heart age between 0 and 5 years greater, 29.3\% $(n=83)$ between 5 and 10 years greater, $24.9 \%(\mathrm{n}=71)+10$ and 15 years greater, and $29.3 \% \quad(n=83) \geq 15$ years greater than their chronological age.

When assessed in relation to QRISK2 risk, two-thirds of low-risk patients and $85.5 \%$ of intermediate-risk patients had heart ages $\geq 5$ years greater than their age. Also, $18.8 \%$ of low-risk patients and $53.6 \%$ of intermediate-risk patients had heart ages $\geq 10$ years greater than their age.

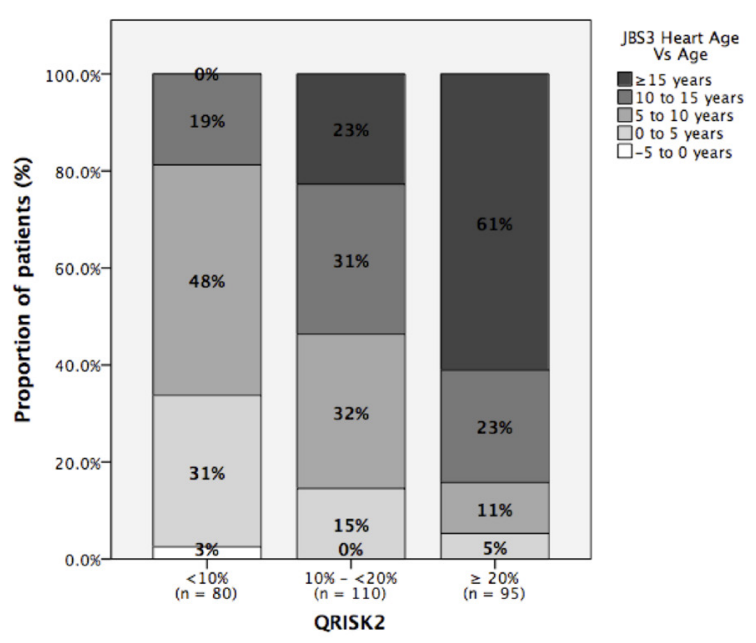

Figure 3 Difference between JBS3 'heart age' and chronological age for cohort and according to QRISK2 10year cardiovascular disease risk category. 


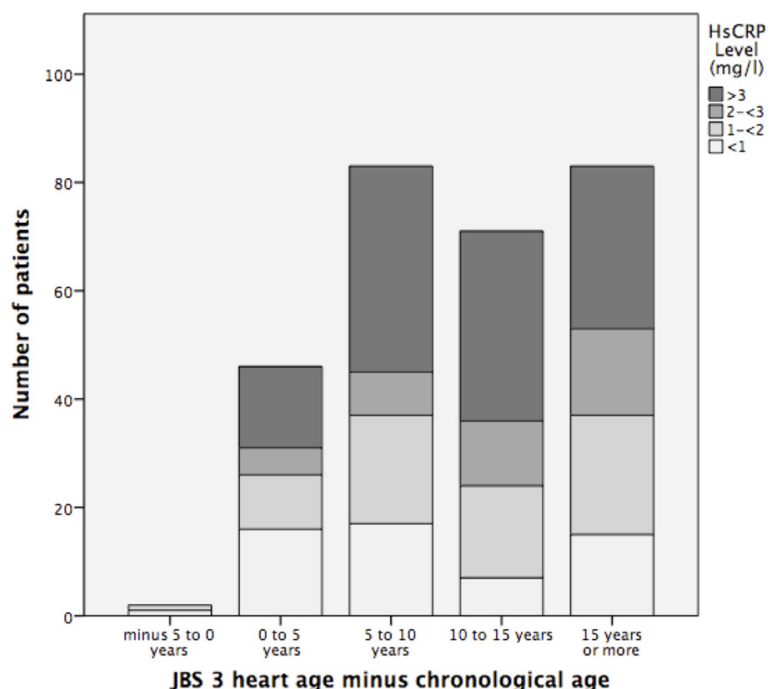

Figure 4 High-sensitivity C(hsCRP) levels according to difference between JBS3 'heart age' and chronological age.

\section{Heart age and hsCRP}

The distribution of hsCRP levels within heart age categories is shown in figure 4. Moreover, 159 of 285 patients without diabetes mellitus or statin treatment $(55.8 \%)$ had elevated heart age and hsCRP levels $\geq 2 \mathrm{mg} / \mathrm{L}$. Of the 80 patients classified as low risk by QRISK2, $63(78.8 \%)$ had both increased heart age relative to age and elevated hsCRP levels $(\geq 1 \mathrm{mg} / \mathrm{L}) ; 40$ of these patients $(50.0 \%)$ had both increased heart age and hsCRP levels $\geq 2 \mathrm{mg} / \mathrm{L}$ (online supplementary table 1).

\section{DISCUSSION}

In this analysis of data from the UK cohort of the EURIKA study of patients over the age of 50 and with at least one major CVD risk factor, free from diabetes and not taking a statin, we found that a third of patients were classified as high risk by QRISK2, the conventional cardiovascular risk calculator used in the UK, and therefore eligible for statin therapy using a conventional $\geq 20 \%$ treatment threshold at the time of the EURIKA study data collection, which occurred between May 2009 and January 2010. ${ }^{16}$ However, given the further lowering of the treatment threshold for initiation of lipid-lowering therapy in the 2014 NICE guidance to an estimated 10-year CVD risk of $\geq 10 \%,{ }^{5}$ we estimate that almost an additional $40 \%$ of untreated patients $\geq 50$ years old with at least one major risk factor would have been eligible for high-intensity statin treatment. Only $\sim 30 \%$ of this population would have been considered low risk and ineligible for statin treatment according to NICE guidance. ${ }^{5}$

The 2014 JBS3 recommendations emphasise the need for greater focus on lifetime CVD risk assessment, given that the majority of cardiovascular events occur in those identified as intermediate risk as assessed by conventional 10-year risk scoring systems. ${ }^{6}$ JBS3 also highlights that short-term risk measures often fail to identify younger patients, especially women, who may be at considerable lifetime exposure to modifiable CVD risk factors but with modest short-term risk scores, and could derive benefit from early risk factor management as part of primary prevention. This has led to the introduction of risk metrics such as 'heart age', incorporated in the JBS3 tool, which aim to quantify this lifetime risk and aid clinical decision-making and communication of risk with patients. Our data demonstrate that in low-risk and intermediate-risk patients with at least one major risk factor, significant proportions have a predicted JBS3 'heart age' of at least 5 and even 10 years or more higher than their chronological ages. While the threshold for considering a heart age to be significant relative to age may not easily be defined in absolute terms, it may be appropriate to consider this measure when considering whether to initiate drug therapy if there is uncertainty or after lifestyle interventions have been unsuccessful.

Results from our analysis demonstrate that a large proportion of patients have clinically relevant elevation in hsCRP levels. This applies across all 10-year risk categories as assessed by QRISK2. Previous work using data from the entire European EURIKA cohort identified similar proportions of patients with elevated hsCRP across risk categories when applying Systematic Coronary Risk Evaluation (SCORE) and Framingham Risk (FRS) Scores for CVD risk assessment. ${ }^{19}$ For clinical decision-making, this has previously been most relevant in patients classified as intermediate risk by conventional methods. ${ }^{20}$ Although not routinely used in the $\mathrm{UK}$, the recent lowering of the threshold for considering initiation of statin treatment by NICE has further weakened the argument for the measurement of hsCRP in intermediate-risk UK patients. Nonetheless, identification of elevated hsCRP still identifies a population for whom a greater absolute benefit of statin therapy might be realised than would otherwise be expected based on their traditional risk factor profile.

The JUPITER trial demonstrated a $44 \%$ reduction in cardiovascular events in patients receiving rosuvastatin $20 \mathrm{mg}$, all of whom had elevated hsCRP levels but a lipid profile that would not warrant statin therapy according to contemporary US clinical guidelines (LDL-cholesterol $<3.4 \mathrm{mmol} / \mathrm{L}) .{ }^{14}$ Indeed, rosuvastatin almost halved major CVD events in those with a 10-year CVD risk of $5 \%-10 \%$ by Framingham Risk Score. A prospective study, conducted in a more general UK population, identified approximately one-fifth of study participants as qualifying for medical therapy under JUPITER criteria who would not be candidates according to established scoring systems, and these subjects had a significantly increased event rate (HR 1.70)..$^{21}$ Our results reveal a considerable proportion of low-risk patients in the UK ( $\geq 50$ years with one conventional risk factor) with elevated hsCRP levels who may therefore be at higher CVD risk than would be predicted based on conventional assessment methods and who may derive greater absolute clinical benefit from statin therapy with greater cost-effectiveness. However, 
these numbers would be smaller than those newly eligible through lowering of the conventional CVD risk score threshold from $\geq 20 \%$ to $\geq 10 \%$.

It may be useful to consider lifetime risk metrics such as heart age in conjunction with biomarkers such as hsCRP to identify those who are both at significant long-term cardiovascular risk based on their traditional risk factors in addition to an elevated level of background vascular inflammation. The results of the recent CANTOS trial demonstrated a significant reduction in cardiovascular events in patients with previous myocardial infarction and hsCRP levels $>2 \mathrm{mg} / \mathrm{L}$ who were randomised to receive monoclonal antibody targeted to reduce serum IL-1 $\beta$ and hsCRP levels without modification of lipid profile. ${ }^{15}$ Although the CANTOS study was in a secondary prevention population, it has added further weight to the independent role of inflammation in atherosclerosis and the use of inflammatory biomarkers in identifying those at risk of future events who may benefit from early intervention. Of note, almost half of patients with a low QRISK2 score within our study population who had a heart age greater than their chronological age also had hsCRP levels greater or equal to the JUPITER and CANTOS threshold of $2 \mathrm{mg} / \mathrm{L}$ and might potentially benefit from a more intensive CVD risk-lowering treatment strategy.

The strengths of the EURIKA study protocol are the standardisation of data collection and the use of a central laboratory for blood analyses (including hsCRP assays). ${ }^{16}$ Furthermore, the inclusion of physicians from a variety of medical specialties and the consecutive selection of eligible patients is likely to have provided a broad representation of such UK patients. It is possible, however, that the brevity of the recruitment period (3 months) may have biased selection towards less healthy, frequent users of healthcare services, who may have higher levels of hsCRP.

In conclusion, our analysis of data from UK patients aged 50 years or more with at least one cardiovascular risk factor has shown that an additional $40 \%$ of untreated patients within this 'at risk' cohort may be eligible for statin initiation following the reduction in the treatment threshold by NICE to $\geq 10 \% 10$-year CVD risk. A considerable number of patients with low or intermediate risk scores had a heart age, as modelled by JBS3, greater than their chronological age and may be at greater lifetime risk than reflected by their 10 -year risk score. Additionally, a large proportion of patients aged 50 years or more with at least one CVD risk factor have elevated hsCRP levels. Measurement of hsCRP in patients classified as low risk in conjunction with lifetime risk metrics such as heart age may further identify a substantial proportion of patients who are at higher risk than might otherwise be predicted by conventional metrics and who may derive greater absolute clinical benefit from statin therapy at greater cost-effectiveness.

\section{Author affiliations}

${ }^{1}$ Western Health, Victoria, Saint Albans, Australia
${ }^{2}$ King's College Hospital NHS Foundation Trust, London, UK

${ }^{3}$ Medical Evidence and Observational Research, AstraZeneca, Madrid, Spain ${ }^{4}$ Cardiology Department, University Hospital of Wales, Cardiff and Vale University Health Board, Cardiff, UK

${ }^{5}$ General Practitioner, Wokingham, UK

${ }^{6}$ Swansea University Medical School, Swansea, UK

Contributors IJ and JPH performed the data analysis and drafted the manuscript. All remaining authors reviewed and provided feedback on the data and contributed to the final version of the manuscript.

Funding The EURIKA study was funded by AstraZeneca. The main study was run by an independent academic steering committee. The authors had full access to all data and had final responsibility for the contents of the manuscript and the decision to submit it for publication.

Competing interests JPH has received fees for participation in review activities for AstraZeneca, is a consultant and has received payment for lecturing, development of educational presentations from Astra Zeneca, is a consultant for Abbott, Pfizer, Roche and MSD and has received fees for lecturing from Abbott, Roche, MSD and Takeda as well as has had accommodation and meeting expenses covered from Abbott. JM works for Astra Zeneca. GK has received payment for manuscript preparations (not including the current one) by the Guidelines Board for management of myalgia in patients taking statins.

Patient consent Obtained.

Ethics approval The study protocol was approved by the appropriate clinical research ethics committees in each participating country. Specifically, the protocol was approved by the following ethics committees: Ethics Committee of Hospital Barmherzige Brüder, Vienna, Austria; Ethics Committee of University Hospital, Ghent, Belgium; National Commission on Informatics and Liberties, Paris, France; Ethics Committee of the Friedrich Alexander-University, Erlangen-Nuremberg, Germany; Scientific Council of University General Hospital of loannina and the National Organization for Medicines (EOF), Greece; Regional Committee for Ethics in Medicine and Research Sor-øst B (REK Sor-øst B), Oslo, Norway; Independent Interdisciplinary Ethics Committee, Moscow, Russia; Clinical Research Ethics Committee of La Paz University Hospital, Madrid, Spain; Ethics Committee of the University Hospital of Linköping, Sweden; Ethics Committee for Ambulatory Clinical Research, Medical Association of Geneva, Switzerland; Research Ethics Committee of Medical Faculty, Gazi University, Ankara, Turkey; Brent Primary Care Trust Applied Research Unit, National Health Service, London, UK.

Provenance and peer review Not commissioned; externally peer reviewed.

Data sharing statement Data underlying the findings described in this manuscript may be obtained in accordance with AstraZeneca's data sharing policy described online (https://astrazenecagrouptrials.pharmacm.com/ST/Submission/ Disclosure).

Open access This is an open access article distributed in accordance with the Creative Commons Attribution Non Commercial (CC BY-NC 4.0) license, which permits others to distribute, remix, adapt, build upon this work non-commercially, and license their derivative works on different terms, provided the original work is properly cited, appropriate credit is given, any changes made indicated, and the use is non-commercial. See: http://creativecommons.org/licenses/by-nc/4.0/

\section{REFERENCES}

1. Allender $S$, Scarborough $P$, Peto V. European cardiovascular disease statistics. European Heart Network 2008;3:11-35.

2. Townsend N, Wickramasinghe K, Bhatnagar P. Coronary heart disease statistics 2012 edition. London: British Heart Foundation, 2012.

3. Authors/Task Force Members:, Piepoli MF, Hoes AW, et al. 2016 European Guidelines on cardiovascular disease prevention in clinical practice: The Sixth Joint Task Force of the European Society of Cardiology and Other Societies on Cardiovascular Disease Prevention in Clinical Practice (constituted by representatives of 10 societies and by invited experts) Developed with the special contribution of the European Association for Cardiovascular Prevention \& Rehabilitation (EACPR). Atherosclerosis 2016;252:207-74.

4. Hippisley-Cox J, Coupland C, Vinogradova Y, et al. Predicting cardiovascular risk in England and Wales: prospective derivation and validation of QRISK2. BMJ 2008;336:1475-82.

5. NICE. Lipid modification: cardiovascular risk assessment and the modification of blood lipids for the primary and secondary prevention of cardiovascular disease 2014. 
6. Boon N, Boyle R, Bradbury K. Joint British Societies' consensus recommendations for the prevention of cardiovascular disease (JBS3). Heart 2014;100(Suppl 2):pp.ii1-ii67.

7. Pearson TA, Mensah GA, Alexander RW, et al. Markers of inflammation and cardiovascular disease: application to clinical and public health practice: a statement for healthcare professionals from the Centers for Disease Control and Prevention and the American Heart Association. Circulation 2003;107:499-511.

8. Emerging Risk Factors Collaboration, Kaptoge S, Di Angelantonio $\mathrm{E}$, et al. C-reactive protein concentration and risk of coronary heart disease, stroke, and mortality: an individual participant metaanalysis. Lancet 2010;375:132.

9. Ridker PM, Hennekens $\mathrm{CH}$, Buring JE, et al. C-reactive protein and other markers of inflammation in the prediction of cardiovascular disease in women. N Engl J Med 2000;342:836-43.

10. Ridker PM, Rifai N, Rose L, et al. Comparison of C-reactive protein and low-density lipoprotein cholesterol levels in the prediction of first cardiovascular events. N Engl J Med 2002;347:1557-65.

11. Danesh J, Wheeler JG, Hirschfield GM, et al. C-reactive protein and other circulating markers of inflammation in the prediction of coronary heart disease. N Engl J Med 2004;350:1387-97.

12. Emerging Risk Factors Collaboration, Kaptoge S, Di Angelantonio $\mathrm{E}$, et al. C-reactive protein, fibrinogen, and cardiovascular disease prediction. N Engl J Med 2012;367:1310-20.

13. Khot UN, Khot MB, Bajzer CT, et al. Prevalence of conventional risk factors in patients with coronary heart disease. JAMA 2003;290:898-904.

14. Ridker PM, Danielson E, Fonseca FA, et al. Rosuvastatin to prevent vascular events in men and women with elevated C-reactive protein. N Engl J Med 2008;359:2195-207.
15. Ridker PM, Everett BM, Thuren T, et al. Antiinflammatory therapy with canakinumab for atherosclerotic disease. N Engl J Med 2017;377:1119-31.

16. Rodríguez-Artalejo F, Guallar E, Borghi C, et al. Rationale and methods of the European Study on Cardiovascular Risk Prevention and Management in Daily Practice (EURIKA). BMC Public Health 2010;10:382.

17. Graham I, Atar D, Borch-Johnsen K, et al. European guidelines on cardiovascular disease prevention in clinical practice: executive summary: Fourth Joint Task Force of the European Society of Cardiology and Other Societies on Cardiovascular Disease Prevention in Clinical Practice (Constituted by representatives of nine societies and by invited experts). Eur Heart $J$ 2007;28:2375-414.

18. JBS3 Risk Calculator. 2018. Available from: http://www.jbs3risk.com/ pages/risk_calculator.htm

19. Halcox JP, Roy C, Tubach F, et al. C-reactive protein levels in patients at cardiovascular risk: EURIKA study. BMC Cardiovasc Disord 2014;14:25

20. Ridker PM, Macfadyen JG, Nordestgaard BG, et al. Rosuvastatin for primary prevention among individuals with elevated highsensitivity C-reactive protein and $5 \%$ to $10 \%$ and $10 \%$ to $20 \%$ 10 -year risk. Implications of the Justification for Use of Statins in Prevention: an Intervention Trial Evaluating Rosuvastatin (JUPITER) trial for "intermediate risk". Circ Cardiovasc Qual Outcomes 2010;3:447-52.

21. Sondermeijer BM, Boekholdt SM, Rana JS, et al. Clinical implications of JUPITER in a contemporary European population: the EPIC-Norfolk prospective population study. Eur Heart $J$ 2013;34:1350-7. 